\title{
Refractive Aspect of Cataracts - Towards Customisation
}

\author{
Pascal Rozot and Jean-Claude Rigal-Sastourne
}

Clinique Monticelli, Marseille

\section{Abstract}

Currently, posterior chamber intraocular lenses (IOLS) correct totally or partially spherical aberrations. In this article we present the visual results of a prospective multicentre study evaluating the Micro AY IOL correcting $0.11 \mu \mathrm{m}$ of spherical aberration, which is a good compromise between optimal vision and a consistent depth of focus. We evaluated 124 eyes that were operated on using bi-manual micro-cataract surgery (BiMICS: 75\%) or co-axial micro-cataract surgery (CoMICS: 25\%) phacoemulsification with insertion of a Micro AY IOL through a 1.8-2.2mm incision. Mean post-operative best corrected visual acuity was $0.97 \pm 0.25$; pre-operative spherical aberrations were $+0.24 \pm 0.13 \mu \mathrm{m}$, and reached $+0.10 \pm 0.11 \mu \mathrm{m}$ post-operatively. There were no complications due to the lens, and photopic contrast sensitivity reached high levels.
\end{abstract}

\section{Keywords}

Phacoemulsification, posterior chamber intraocular lens, spherical aberrations, contrast sensitivity, depth of focus

Disclosure: The authors have no conflicts of interest to declare.

Received: 9 November 2009 Accepted: 3 December 2009 DOI: 10.17925/EOR.2009.03.02.80

Correspondence: Pascal Rozot, Clinique Monticelli, 88 rue du Commandant Rolland, 13008 Marseille, France. E: pascalrozot@sfr.fr

Whether bi-manual or co-axial, the microincision phacoemulsification techniques of today allow for a neutral cylinder, thus reducing high-degree aberrations (in particular the coma type). The hydrophilic acrylic intraocular lenses (IOLS) are more compliant as they are inserted through a minimum $1.6-1.8 \mathrm{~mm}$ incision, whereas the hydrophobic IOLs are inserted through $2-2.2 \mathrm{~mm}$ incisions but generate fewer secondary cataracts. The general idea of aspherical IOLS is to compensate all or part of Z4 types of positive corneal spherical aberrations (SAS) with a properly adapted optic. On average, corneal spherical aberrations are $+27 \mu \mathrm{m}$ for a $6 \mathrm{~mm}$ pupil, which corresponds to a mean $\mathrm{Q}$ co-efficient of -27 . In order to choose the correct asphericity profile, the patient's corneal profile must first be studied by measuring not only the Z4 and Q factor but also his/her keratometry data. It is necessary to know the levels of asphericity of the different IOLs on the market and finally to take into consideration the power of the implant.

Current corneal topographic devices measure the value of the $\mathrm{Q}$ factor directly, as well as the spherical distortions of each cornea. Historically, the first implants totally compensated corneal SAs, but over the last few years we have been aiming at only partial correction of these SAs in order to achieve the best visual result, which we know is obtained with approximately $+0.10 \mu \mathrm{m}$ total residual SA. ${ }^{1}$ As correction of SAS also provides a better contrast sensitivity in photopic and mesopic conditions, the main clinical interest in correcting spherical SAs is specifically seen in mesopic and scotopic conditions with a pupil dilated at approximately $6 \mathrm{~mm}$, which improves the ability of night driving. With this in mind, it can be interesting to systematically carry out corneal topography on all young patients having undergone cataract surgery in order to optimise their vision in these conditions, ${ }^{2}$ because we know they will be driving at night.
On the other hand, depth of focus induced by implants correcting SAs can be reduced, but this is not an established fact as different studies have sometimes given conflicting results.

The results of a prospective multicentre study carried out by four surgeons concerning a microincision implant (see Figure 1) offering a $-0.11 \mu \mathrm{m}$ asphericity through a $1.8 \mathrm{~mm}$ incision (Micro AY implant made by PhysIOL laboratories) are presented here, covering 124 cases of senile or pre-senile cataracts. The population was made up of $54 \%$ females and $46 \%$ males, of whom $23 \%$ presented cortical type of cataract, $58 \%$ nuclear cataracts and 19\% subcapsular cataracts. Mean age was $73.5 \pm 8.3$ years (range 51-89 years). Topical anaesthesia was used in $96 \%$ of cases with $100 \%$ clear cornea incisions. The incision was temporal in $59 \%$ of cases, $27 \%$ on the most arched meridian and superior in $3 \%$ of cases. The bi-manual technique was used for $92(74.2 \%)$ of patients. The mean incision for phacoemulsification was $1.41 \mathrm{~mm}$ for all of the interventions and $1.95 \mathrm{~mm}$ for implantation.

Mean endothelial cell loss was 6\%. Of 101 cases, uncorrected visual acuity was $0.72 \pm 0.22$ at three months and best corrected visual acuity (BCVA) was $0.97 \pm 0.25$ (see Figure 2). Post-operative spherical equivalent was $-0.36 \pm 078 \mathrm{D}$ (compared with $0.51 \pm 2.31 \mathrm{D}$ preoperatively). Pre-operative cylinder was $-0.34 \pm 0.87$ and reached $-0.14 \pm 0.71 \mathrm{D}$ post-operatively. In aberrometry, the pre-operative corneal Z4 were $+0.24 \pm 0.13$ (from -0.09 to +0.31 ) and reached $+0.10 \pm 0.11 \mu \mathrm{m}$ (from -0.12 to +0.24 ) post-operatively. No complications were due to the implant: there were no problems during insertion with the PhysIOL injector, no decentration, no IOL deposits and no posterior capsule early opacity (see Figure 3). Results for contrast sensitivity are presented in Figure 4; depth of focus (DF) was compared with a series 


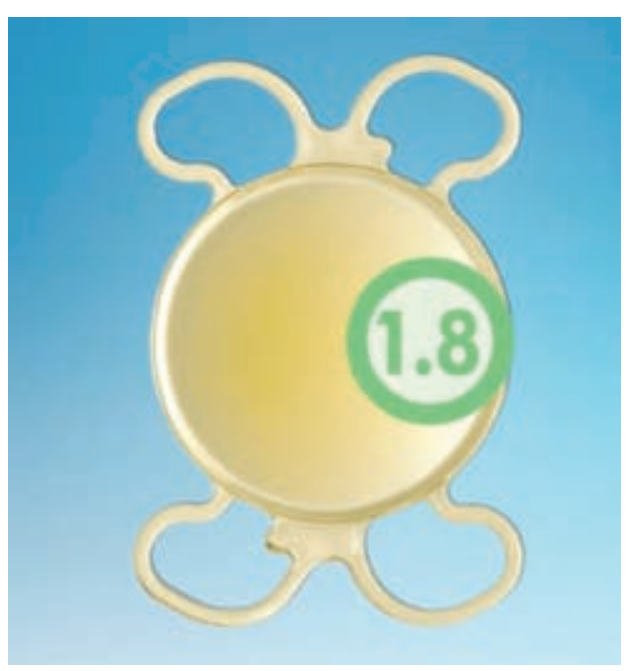

Figure 2: Best Corrected Visual Acuity

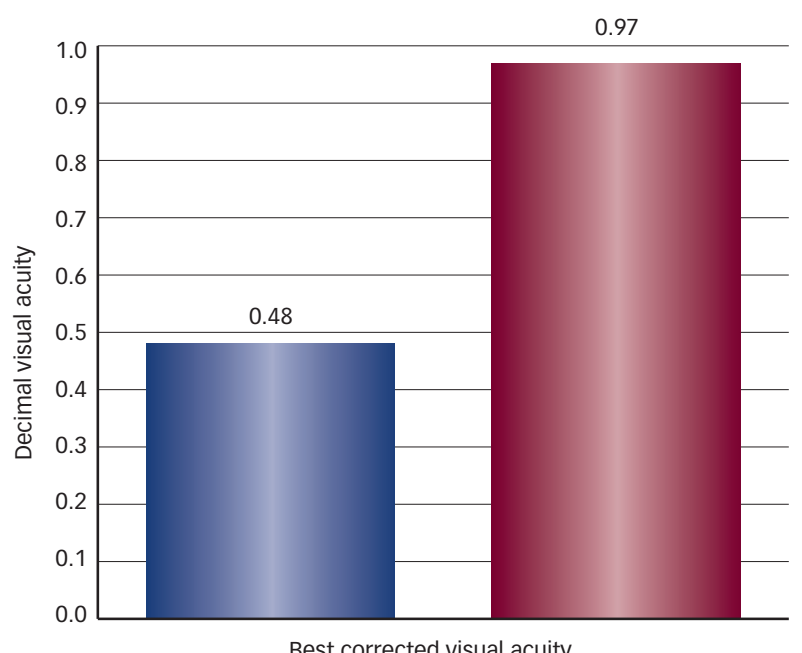

II Pre-operative $(n=124) \quad$ II 3 months $(n=101)$

of spherical lenses, confirming that there was a slight decrease of DF (see Figure 5) but to a lesser extent than in full correcting SA IOLS.

This study confirmed that the refractive approach to SAs of the cataract improved the pseudophakic's quality of vision. On the whole, customising the correction of SAs will depend on the corneal topography in certain cases: young adults driving at night, patients with large pupils, those with diffractive multifocal IOLs and patients with particular corneal profiles, especially those having undergone refractive surgery. Indeed, it has been pointed out that a mean partial correction of SAs in all of the studies shows an increase in contrast sensitivity with implants having an aspherical optic as long as the implant is correctly centred. However, correcting spherical aberrations will always come after the correction of spherical and cylindrical ametropia relevant to the quality of surgery and the precision of biometrical calculations.

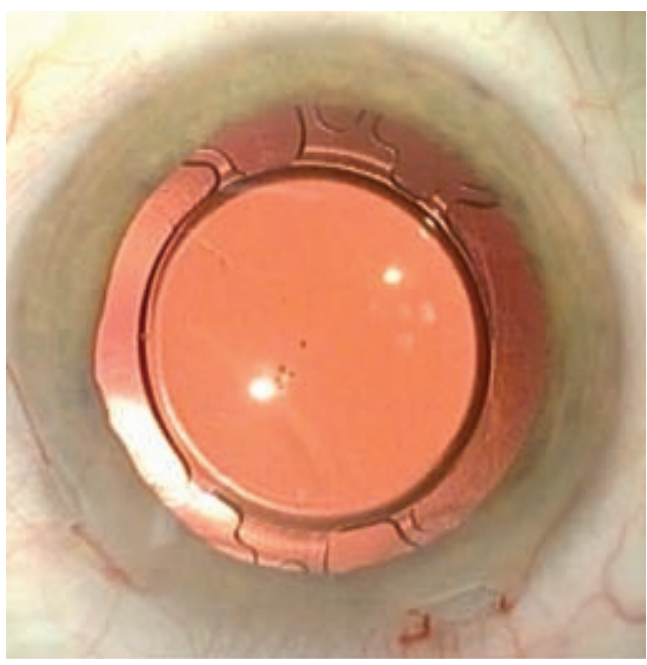

Figure 4: Contrast Vision (ETDRS)

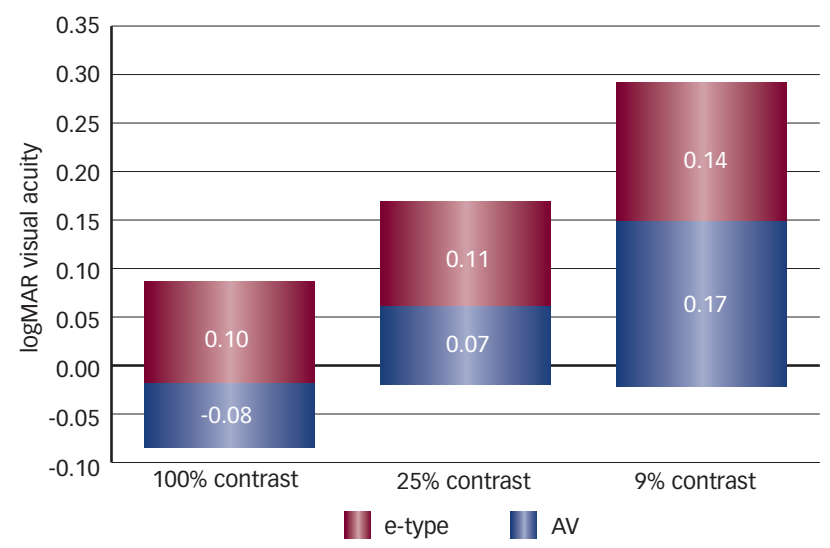

$A V=$ arteriovenous; $E D T R S=$ Early Treatment Diabetic Retinopathy Study.

Figure 5: Comparison of Defocus Curves

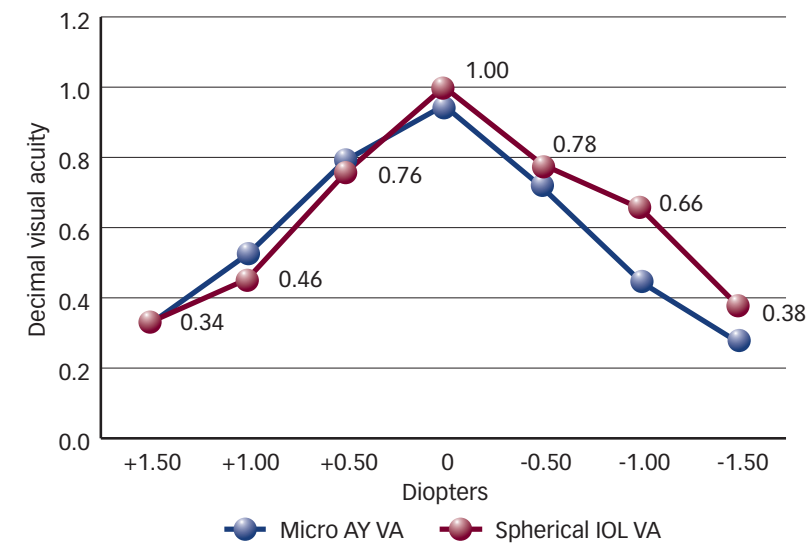

101 = intraocular lens; $V A=$ visual acuity
1. Levy Y, Segal O, Avni I, Zadok D, Ocular higher-order aberrations in eyes with supernormal vision, Am J ophthalmol, 2005;139(2):225-8.
2. Nochez Y, Favard A, Majzoub S, Pisella PJ, Measurement of corneal aberrations for customization of intraocular lens asphericity: impact on quality of vision after micro-incision cataract surgery, Br J Ophthalmol, 2009; (Epub ahead of print). 